\title{
ACUTE KIDNEY INJURY
}

\section{Effect of perioperative aspirin and clonidine on AKI}

New findings, presented at ASN

Kidney Week, show that perioperative administration of aspirin or clonidine does not reduce the risk of acute kidney injury (AKI) among patients undergoing noncardiac surgery. "These results support the practice of halting aspirin use in the perioperative period among patients who take aspirin as part of a long-term regimen, as they do not derive any benefit from this therapy, which increases the risk of bleeding," says researcher Amit Garg.

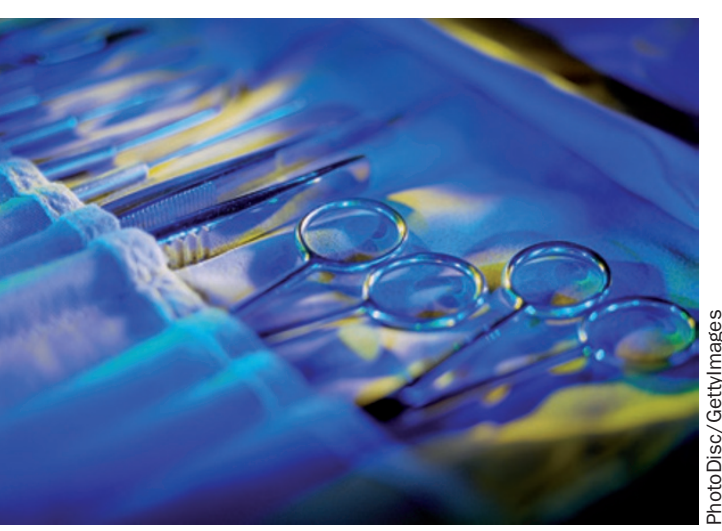

AKI, a common complication of surgery, is associated with poor outcomes and high health-care costs. Promising data from preclinical and clinical studies suggested that the common antiplatelet aspirin or the centrally acting $\alpha 2$-adrenergic agonist clonidine might reduce the risk of AKI.

To investigate the effects of aspirin and clonidine on AKI, Garg and colleagues performed a substudy of the POISE-2 trial, by randomly assigning 6,905 patients undergoing noncardiac surgery across 88 centres to receive aspirin $(200 \mathrm{mg}$ ) or placebo $2-4 \mathrm{~h}$ before surgery and then aspirin $(100 \mathrm{mg}$ ) or placebo daily up to 30 days after surgery, or to receive oral clonidine $(0.2 \mathrm{mg})$ or placebo $2-4 \mathrm{~h}$ before surgery and then a transdermal clonidine patch ( $0.2 \mathrm{mg}$ per day) or a placebo patch that remained until $72 \mathrm{~h}$ after surgery.

The researchers found no difference in the risk of AKI among patients receiving aspirin or clonidine versus placebo. They performed post hoc analyses to investigate whether the interventions could cause harm.
"In POISE-2 we demonstrated that aspirin and clonidine increase the risk of major bleeding and hypotension, respectively," explains Garg. "In this substudy, such bleeding or hypotension was associated with a greater risk of AKI. There was also some suggestion that each intervention increased the risk of severe AKI; however, the results for this secondary outcome need to be interpreted cautiously."

The researchers say their findings suggest that future interventional trials for AKI in the surgical setting should focus on interventions that target pathways other than platelet aggregation or $\alpha 2$-adrenergic signalling. "Interventions that place emphasis on the prevention of perioperative bleeding and hypotension may prove beneficial," suggests Garg.

Susan J. Allison

Original article Garg, A. X. et al. Perioperative aspirin and alonidine and risk of acute kidney injury: a randomized clinical trial. JAMA doi: 10.1001/jama.2014.15284 